THE TRANSACTIONS OF THE ROYAL INSTITUTION OF NAVAL ARCHITECTS

Part A - International Journal of Maritime Engineering

Format for submitted papers and technical notes

This document is formatted in the convention required for all papers/technical notes.

\title{
Experimental investigation into factors affecting the transient flow of fluid through an orifice in realistic conditions
}

(DOI No: 10.3940/rina.ijme.2015.a1.???)

C.D.Wood, University of Southampton, UK, D.A.Hudson, University of Southampton, UK, M.Tan, University of Southampton, UK, A.J.Sobey, University of Southampton, UK and Y.Wang, University of Southampton, UK

\section{SUMMARY}

During operations, damage can occur with a resulting ingress or egress of fluid. The incoming water affects the reserve buoyancy and it can also change stability and hull girder loading. During a flooding event it is vital that the flow through the damaged orifice and the movement of floodwater around the structure can be predicted quickly to avoid further damage and ensure environmental safety. The empirical measure coefficient of discharge is used as a simplified method to quantify the flooding rate. In many internal flow applications the coefficient of discharge is estimated to be 0.6 but recent research shows that it can vary considerably when applied to transient flooding flows. This paper uses an experimental setup to investigate how changes to the orifice edges and position within the structure affect the flow. It is then used to investigate the coefficient in a more realistic scenario, a static compartment in waves.

\section{INTRODUCTION}

During maritime operations accidental damage can allow fluids to flow into or out of the structure. The damage can occur for many different reasons but it is vital for the environment and safety that the impact of the damage can be quickly and easily determined. A simplified understanding of how the variables associated with the orifice affect the flow could be used to provide guidance to crews in how to best react to damage scenarios and aid salvage.

Previous studies have investigated the behaviour of damaged vessels concentrating on the flooding of ferries such as Spouge (1986) who found it only took minutes for capsize for transient asymmetric flooding with further examples using time domain simulation methods performed by Dand (1988),
Vassalos and Turan (1994), and Chang and Blume (1998). These results show that simulation of the ship's flooding and capsizing time history is possible but the time requirements are inhibitive to emergency response usage.

In practice an initial estimation of flooding rate, the coefficient of discharge, $C_{d}$, is commonly used. If the value of the $C_{d}$ is known, an assessment of a damage scenario can be made quickly. Existing research on flooding rates is based on steady state flow through circular orifices with different thicknesses of the orifice plate, ratio of orifice diameter to external geometry and local changes of geometry within the circular orifice. Existing research into progressive flooding identifies that discrepancies exist between assumed and measured values of discharge coefficient Ruponen (2007) but 
little research has been conducted in an attempt to predict these values; it is rare that real damaged orifices are of a regular shape and smooth. Some early experiments conducted by Smith and Walker (1923) found the coefficient of discharge varies from 0.67 for a $2 \mathrm{~cm}$ orifice to 0.614 for a $6 \mathrm{~cm}$ orifice with heads above $1.2 \mathrm{~m}$. Whilst a number of studies investigated $\mathrm{C}_{d}$ few of these were specific to flooding of compartments. Recently, Ruponen (2006, 2010) completed a series of experiments to measure the flooding rate of a box-shaped barge generating a range of coefficients of discharge. These tests showed a fluctuation of between 0.720.83 for the coefficient of discharge in different types of flooding scenarios such as broken pipes and staircases. A range of damage sizes were also investigated from $20 \mathrm{~mm} \times 20 \mathrm{~mm}$ to $100 \mathrm{~mm} \times 100 \mathrm{~mm}$ where the $C_{d}$ value decreases as the opening size increases. This trend was again experimentally shown by Smith (2009) where a hydraulic model was used to measure the discharge coefficient of different damage geometries. The model was forced at constant vertical velocities and the discharge coefficient was calculated for each velocity. The results show a strong sensitivity to the orifice size and flow direction, ingress/egress, and less sensitivity to the velocity of the model. In addition, the jet velocity also decreased when the orifice size increased. Hearn et al (2008) calculated the relevant coefficients of floodwater to analyse flooding flow. Wood et al. (2010) experimentally determined the $C_{d}$ values for 10 orifices with different shape and areas and compared the results with CFD simulation. These results show that as the area gets smaller the $C_{d}$ value decreases and the shape has a large effect on the $C_{d}$ value. Further research by Wood (2013) showed that the $C_{d}$ value was time dependent during the flooding process. $C_{d}$ values varied from 0.3 to over 1 depending on when the measurements were taken. In addition, averaged transient flooding $C_{d}$ varied from 0.53 to 0.74. Li et al. (2013) performed experiments that highlighted the change in coefficient of discharge between flat orifices and those containing more realistic petalled edges. This work was extended by Wang et al. (2015) to investigate the same effects looking at the flow between water and oil. These investigations focus on static water but recent research shows the importance of movement and wave motions. Domeh et al. (2015) investigate the effect of compartment permeability on the flooding of damaged vessels. This study shows that for stationary vessels the permeability and orifice size had limited effects. However, at forward speed the permeability and orifice size had a much larger effect on the stability of the vessel. The benefit of the addition of motion was also shown by Suyuthi et al. (2013).

A number of previous studies have shown the effects of orifice size and shape on the fluid flow with different studies have used a combination of experiments and CFD to investigate these effects. Recent studies have investigated the coefficient discharge in the context of more realistic scenarios. To continue this trend this paper is comprised of two investigations; the manner in which the edges of the orifice and its position within the structure affect the flow rate, and the effect of passing waves on orifice flow into a compartment which are performed to gain a better understanding of the coefficient of discharge for more practical applications.

\section{EXPERIMENTAL SETUP}

A fully venting floodable compartment experimental rig has been constructed out of aluminium and clear acrylic tube, with inner diameter of $190 \mathrm{~mm}$ diameter and internal length of $674 \mathrm{~mm}$, as shown in figure 1 . The floodable cylinder was placed into the Lamont towing tank, $30 \mathrm{~m}$ long, $2.44 \mathrm{~m}$ wide and $1.012 \mathrm{~m}$ deep, so that the depth of the damage orifice was $0.3 \mathrm{~m}$. Waves were generated using a SEASIM wave maker, a three paddle pneumatic wave making device. The wave period was controlled by a hexadecimal input and wave height is controlled by a gain dial. The wave probe arrangement has been modified to provide two free surface height measurements, one from inside the compartment and the other from outside.

For the experiments in waves three wave heights, $14 \mathrm{~mm}, 24 \mathrm{~mm}$ and $31 \mathrm{~mm}$, and seven wave time periods were used that cover the operating range of the wave makers: $0.5 \mathrm{~s}, 0.6 \mathrm{~s}, 0.75 \mathrm{~s}, 0.9 \mathrm{~s}, 1 \mathrm{~s}, 1.25$ $\mathrm{s}$ and $1.5 \mathrm{~s}$. Flooding experiments were conducted for combinations of these conditions using small, medium and large circular orifices, with diameters of $40 \mathrm{~mm}, 60 \mathrm{~mm}$ and $80 \mathrm{~mm}$, as used in the previous experiments. Experimental water level measurements were taken using wave probes inside the cylinder. During the transient phase of flooding, the oscillating wave pressure caused little change in the flooding rate. Therefore, time to flood was determined using least squares fitting of the experimental data.

A time dependent flooding model developed in Wood (2013) is used for comparison to the experimental data. The pressure difference across the orifice is calculated externally using linear airy wave pressure and internally using the quasi-steady hydrostatic head calculated from the instantaneous 
height of the flood water in the compartment. Sloshing and swirling motions are approximated as superposed sinusoidal surface elevations with amplitudes and phase angles tuned to fit the experimental observations.

\section{CALM WATER FLOODING RESULTS}

The sensitivity of the discharge coefficient to changes in the orifice are measured experimentally. The flow rate through an orifice is tested using an empty cylinder placed inside of a larger tank of water. The water inside the cylinder is evacuated using air pressure. The pressure inside the cylinder is then released and flooding commences.

\subsection{IRREGULAR SHAPES}

To retain the controlled nature of experimentation, rather than using random irregular shapes, fractals have been used to add perturbations onto regular shape edges. The irregularities of this mathematical family of shapes have been applied to fields such as turbulence and cloud formation and here these irregularities are used to approximate the shape of real damage orifices.

The shapes with the fractal perturbations were drawn in CAD package, Solidworks. These fractals were $2^{\text {nd }}$ order; the addition of further fractals only served to create detail that is too small for practical modelling. Figure 2 shows the outlines of the two irregular shapes tested. Both the hexagonal damage and pentagonal damage were scaled in three different sizes with equivalent orifice areas to the circular orifices and this gave comparable results to the regular shape study. The point at which the coefficient of discharge is calculated makes a large difference to the final value for a transient flooding case. For the results presented here the time to flood was taken as the first point at which the smoothed free surface height measurement crossed the flooded point, sloshing is smoothed out as per the definition of time to flood used in the initial transient flooding study. Each experiment was repeated five times using both wave probes and an average was taken to give a mean time to flood.

Using the time to flood, the transient discharge coefficient was calculated as per the methodology in Wood (2013), and the results are listed in Table 1. The results are grouped into orifice areas, where the notation for the fractal shapes denotes the size and number of sides from the donor regular shape, e.g. Fractal S5 has the smallest area and was created using a pentagon.
The greatest variation in discharge coefficient was observed for the small orifices, a result also found for the regular shapes, for which maximum and minimum discharge coefficients were measured. The trend indicated that for small orifices, flow features developed due to the surface roughness led to greater energy absorption and reduced flow rate. As the orifice areas were enlarged, the effect appears to diminish as the development of flow features were hindered due to a combination of reduced time for them to establish, the presence of the compartment wall, or other flow features in close proximity.

\subsection{ORIFICE EDGE PROFILE}

Damage can occur from either internal or external sources which give rise to directionality of the orifice edge profiles, similar to convergent or divergent nozzles. An additional case has been run with a rounded convergent-divergent profile for completeness. For initial assessment of the effects of this directionality in the damage edge profile, three differing orifice edge layouts were tested. The variation of discharge coefficient due to orifice edge geometry is not a new topic of research, the effects are well documented in the field of steady flow through sharp edged circular orifices, but these trends require interrogation for differences occurring due to the unsteady flow regime. Three orifice edge profiles have been tested and compared to existing data of perpendicular orifice edges using the circular damage shape. The edges are shown in Figure 3.

The results of this sensitivity study are shown in Table 2, where orifice area is shown as the minimum cross sectional area presented to the flow.

Whilst the area of the convergent edge section was smaller than the other edges, the order of magnitude was close to that of the small circle. The convergent section was shown to increase flow rate through the orifice, which was consistent with available data of steady discharge coefficients (Miller, 1978). The divergent and rounded orifice edges have cross sectional areas comparable to the medium circle orifice. The results show that a divergent edge profile resulted in a lower flow rate whilst the rounded orifice edge results in a substantial increase in discharge coefficient. It is the authors' belief that orifice edges that conform to the shape of the stream-tube of a typical orifice will result in a reduced contraction downstream, hence higher discharge coefficient. This has potential implications for flooding models in the steady state phase of the flooding regime, as 
different discharge coefficients will be required for ingress and egress phases of flow for the same orifice.

\subsection{ORIFICE OFF CENTRE LOCATION}

Damage orifices are rarely equidistant from all edges of a structure or the compartment behind it and this introduces asymmetry, in addition to the complications of irregular orifice shape. Two additional orifice plates have been used to provide indication of flooding rate sensitivity to orifice location on the orifice plate. The baseline for comparison is made using the centrally located small circle orifice plate. Flooding times are the mean of five runs using two wave probes located on the inside wall of the floodable compartment; these results are shown in Table 3.

Flooding time varied between these cases by $0.41 \mathrm{~s}$. Where the $0 \mathrm{~mm}$ displacement case was the baseline, and corresponded with a $1 \%$ increase for the $13.75 \mathrm{~mm}$ displacement and a $3 \%$ reduction for the $27.5 \mathrm{~mm}$ case. Due to the design of the flooding compartment rig, testing of orifice flow where the orifice is adjacent to the wall was not possible. This indicated discharge coefficient was only slightly dependent on the orifice location within the parameters investigated.

\section{FLOODING IN WAVES}

A ship in a seaway is subject to motions on waves leading to an oscillation of the pressure immediately outside the orifice. The pressure difference that drives flooding across the orifice is a function of damage depth relative to the free surface, pressure due to the local velocity of the fluid, vessel wave encounter frequency, wave amplitude and vessel motion response, particularly in heave. The effect of a time dependent pressure field, due to waves, has been investigated in two stages; the effect of waves on transient flooding rates, and steady state ingress and egress flow rates. Due to the difficulties of modelling this effect computationally experiments are used to demonstrate the effects of the waves. This is performed using the same experimental flooding compartment equipment as used for the first experiments. Surface time histories were measured during both transient flooding and the flooded steady state region to monitor ongoing internal free surface oscillations excited by the external waves. Figure 4 shows the averaged flooding time for the medium circular orifice, $60 \mathrm{~mm}$ diameter, in waves, with different wave amplitudes and wave periods. In general, a reduction in average flow rate was observed with increasing wave period and amplitude, the average flow rates reduced by a common gradient with increasing wave period. This behaviour manifests in Figure 4 as a general increase in time to flood with increasing wave period.

The largest wave amplitudes yielded the lowest flooding rates, although it was difficult to obtain a trend for the 14 and $24 \mathrm{~mm}$ cases in relation to each other due to the abnormalities seen in the $14 \mathrm{~mm}$ results. It is the authors' belief that the pressure oscillations at the orifice disrupted the energy absorbing flow features and interacted with the unsteady features that drove sloshing inside the compartment. From the comparison of modelling and experimental data in Figure 5, it has been deduced that the reduction in flow rate is not due to wave pressure fluctuations reducing flooding rate and therefore, it must be an effect included within the discharge coefficient. The first plot is the calm water model, solid black line, which has been compared to a flooding in waves model, broken black line, using the calm water discharge coefficient, $\mathrm{C}_{\mathrm{d}}=0.738$. No appreciable difference was made to the time to flood purely as a consequence of the pressure fluctuations from the waves. The reduced discharge coefficient calculated from the averaged time to flood from experimental data was applied to the model and compared to one set of experimental data, and where the smoothing of sloshing was taken into account, the results agree well. The discharge coefficients calculated from the series of experimental data are shown in Table 4.

Free surface height fluctuations within the compartment are in part due to ingress and egress of flood water, and also sloshing and swirl motions. A wave period of $0.5 \mathrm{~s}$ closely corresponds to the observed dominant frequency of sloshing in the compartment and flooding times for this particular wave period depart from an otherwise linear trend as shown in Figure 4. Figure 5 shows a comparison of flooding models in calm water andin waves with experimental results from flooding in waves. Experimentally determined discharge coefficients calculated for calm water and flooding in waves are applied and it is shown that the influence of the transient pressure field at the orifice due to waves play little part in the reduction of flooding rates calculated from results in Figure 4, therefore, the reduction is due to viscous effects and must be applied in the discharge coefficient.

Free surface movement due to ingress and egress is shown in Figure 5. Additional free surface 
movement due to sloshing and swirl of the internal free surface was included by the superposition of linear oscillations with the appropriate frequencies, amplitudes and phase differences. Internal free surface motions in the experimental data were dominated by lateral sloshing with an amplitude of $13 \mathrm{~mm}$ and swirl motion amplitude was $3 \mathrm{~mm}$. Wave time period was $1 \mathrm{~s}$ and wave amplitude 24 $\mathrm{mm}$. The model solution departed from the experimental measurements during the transient phase which was dominated by the collapse of the jet and the unsteady phenomena that drive the sloshing motions. These transient phenomena peak when the floodwater level reached half of the total floodable volume. This model does not account for breaking waves or other small waves travelling on the internal free surface as a result of the jet collapse, which manifested as small discrepancies during the transition to steady state ingress and egress at approximately $4 \mathrm{~s}$.

Figure 6 is a plot of free surface oscillations for varying wave amplitudes of constant wave time period, $\mathrm{T}=1 \mathrm{~s}$ and it is used as an example of the steady state oscillations of the internal floodwater height following the transient phase of flooding - a combination of the ingress and egress of flood water and free surface sloshing and swirling motions. It can be observed that the peak compartment oscillation amplitudes were not proportional to wave amplitude; a reduction in motion amplitude is seen with increasing wave amplitude. Two frequencies of sloshing, $2.15 \mathrm{~Hz}$ and $1.075 \mathrm{~Hz}$, were common to all the flooding runs. The dominant frequency of the sloshing was at $2.15 \mathrm{~Hz}$, which is the lateral sloshing component and is near the natural frequency for the compartment water depth of $0.3 \mathrm{~m}$. Once flooding had ceased, motions of the internal free surface settle as the magnitude of the sloshing amplitude diminished over time.

In the comparison of free surface motion response to varying wave amplitude, motion amplitude decay rates run parallel, starting at $10-15 \mathrm{~mm}$ and decaying to 5 - $10 \mathrm{~mm}$ over 5 seconds. As observed in the transient flooding experiments, the combination of ingress and egress, and the free surface motions, can be seen to cause interfering fluctuations in the free surface height history at a discrete location; as a result, the oscillations vary by between $2-6 \mathrm{~mm}$.

Steady state oscillations were measured for varying wave time period whilst wave amplitude was kept constant at $24 \mathrm{~mm}$. Free surface motion amplitude ranged from $5-15 \mathrm{~mm}$, where the largest amplitudes occurred for cases where the period of the wave was close to, or a multiple of, the resonant period of sloshing motions, 0.465 or $0.93 \mathrm{~s}$. Figure 7 contains the results for a $0.5 \mathrm{~s}$ wave time period flooding experiment, where the sloshing amplitudes were disproportionately larger than the other wave frequencies. It is anticipated that excitation of the resonance frequency gave rise to the larger free surface motions within the flooding compartment. These oscillations can enhance, or hinder, the energy absorption by flow features that modify the orifice flow rate. In cases with larger sloshing amplitudes, the averaged flooding rates were higher. It is author's belief that the establishment of a stable vena contracta must therefore be intermittently disrupted, thus reducing the energy absorption by the flow feature. The amplitude of wave induced free surface oscillations increased with wave time period as the flood water went through ever extended periods of egress. At higher frequencies, relatively little fluid passes through the orifice as wave passes. Based on the findings from the experimental data, and the subsequent comparison to model results, the dominant free surface oscillations changed from sloshing and swirl at high wave frequencies, to compartment floodwater volume oscillations at low wave frequencies. Sloshing amplitude decayed with time in the experimental data as the damping is anticipated to be from wall friction and fluid viscosity. The flooding model does not include any representation of damping, sloshing amplitudes continue at a steady state.

Whilst the model provides a close approximation during the transient phase, the use of the transient discharge coefficient during the steady state flooding stage resulted in error. This was particularly noticeable as an over prediction of flow rates for larger wave periods. For a wave period of $1.5 \mathrm{~s}$ the over prediction in height was in the order of $1 \mathrm{~mm}$, or $\sim 8 \%$ of the total amplitude. The Reynolds number for the flow during the steady state regime was under the threshold value for discharge coefficient scaling limits. To investigate the real world physics with more fidelity a larger floodable compartment is required, this will maintain the discharge coefficient scaling criteria of $\mathrm{Re}>4000$ for a greater period of the flooding time.

\section{DISCUSSION}

Validity of industry standard methods for calculating flooding rate is dependent on the selection of discharge coefficient. Currently an 
approximation value, 0.6, of $C_{d}$ is used, however, this research has reaffirmed that this is not an appropriate assumption in transient flooding cases. What is needed is a prediction method for discharge coefficient for an arbitrary damage orifice.

Novel research has been conducted in the area of transient orifice flow. An experimental study has been carried out to investigate nine cases of transient flooding with the aim of investigating the effects of shape and size. Repeatability of all recorded data is confirmed. The uncertainty in the measured time to flood is $2.9 \%$ for large damage cases and $1.3 \%$ for the smaller damage cases. However it is recognised that measurements taken during the flooding process may be subject to greater error.

Three orifice flow experiments have been conducted in the context of flooding rates in damaged ships; the effect of irregular edges on a damage orifice, non-uniform orifice edge profiles and displacement of a damage orifice from the centre of a damaged panel. Irregular edges on a damage orifice have been shown to absorb more energy from the incoming flow which results in a lower flooding rate. This effect is more prominent for small orifices, where energy absorbing flow features were not hindered by proximity of the wall. Flow through the five sided fractalised shape appeared to be slowed more than that of the hexagonal shape, despite the presence of a larger number of irregular features on the latter. An orifice edge profile survey revealed that any convergence in the edge profile, with respect to the flow direction, results in an increase in discharge coefficient compared to the uniform, or divergent, edge profiles. The effect of displacing the orifice from the centre of the orifice plate was relatively small in comparison to the other sensitivity studies. However, the variation in parameters used in these sensitivity studies was limited and these trends may not hold for all flooding situations.

A series of experiments to determine the effect of waves on transient orifice discharge coefficient has been conducted. The results have been used to model flooding in waves with the inclusion of free surface motions excited during the flooding process. The discharge coefficient has been shown to reduce with increasing wave amplitude and wave time period. The mechanism does not appear to be directly due to the fluctuating pressure difference across the orifice, but due to a viscous or turbulent mechanism which must be accounted for within the discharge coefficient. Free surface motions other than those due to the change in flood water volume, were observed. In order to assess the discharge coefficient during steady state ingress and egress it has become necessary to identify the frequencies and amplitudes of the additional free surface motions. Experimentally, sloshing was observed for all orifice shapes and sizes. A mixture of swirl and side to side sloshing was witnessed during experiments, similar to observations made by Abramson et al. (1966) of sloshing modes in a vertical cylinder. This behaviour is limited to the case of partially filled vertical cylinders; however, regardless of the geometry the unsteady excitations exist and could have an impact on the directions of pressure forces on a flooded compartment. The frequency of the dominant mode of sloshing occurred at approximately $2 \mathrm{~Hz}$, and therefore was independent of damage size. Using the depth of the pressure head as a length scale gave a Strouhal number of 0.23 and was consistent for typical low frequency vortex shedding behaviour; further study would be required to fully understand this phenomenon. Lateral sloshing was the dominant mode of free surface motion in the cylinder, at an orifice depth of $0.3 \mathrm{~m}$ with a frequency of $2.15 \mathrm{~Hz}$. Swirl motions were excited to a lesser extent compared to lateral sloshing and have a frequency of $1.075 \mathrm{~Hz}$. Free surface motion due to ingress and egress became more prevalent with increasing wave time period; becoming the dominant mode in the $1.5 \mathrm{~s}$ wave period cases. Both lateral sloshing and swirl were excited during the flooding process and subsequently decay due to wall friction and fluid viscosity until the free surface settled. With continued wave excitement, motions due to ingress and egress continued in a periodic manner. Ingress and egress of floodwater has been modelled using the mean discharge coefficient during the flood. Largely this was found to give acceptable results; however, for longer wave periods this was found to over predict the flow rates and give larger free surface motion amplitudes due to ingress and egress.

\section{CONCLUSIONS}

Coefficient of discharge is used to help determine the severity and the risks associated with damage to structures in water. Whilst this is the case many applications still use a standard value to estimate the flow rate. In this study a number of variables are investigated to determine the effects on the coefficient of discharge. It is shown that flow through the five sided fractalised shape appeared to be slowed more than that of the hexagonal shape, despite the presence of a larger number of irregular 
features on the latter. An orifice edge profile survey revealed that any convergence in the edge profile, with respect to the flow direction, results in an increase in discharge coefficient compared to the uniform, or divergent, edge profiles. The effect of displacing the orifice from the centre of the orifice plate was relatively small in comparison to the other sensitivity studies. Discharge coefficient has been shown to reduce with increasing wave amplitude and wave time period. Ingress and egress of floodwater has been modelled using the mean discharge coefficient during the flood. Largely this

\section{REFERENCES}

Abramson, H. Chu, W.-H. \& Kana, D. (1966). Some studies of nonlinear lateral sloshing in rigid containers. NASA Contractor report CR-375.

Chang, B.-C. and Blume, P. (1998). Survivability of Damaged Ro-Ro Passenger Vessels. Schiffstechnik - Ship Technology Research, Vol.45, pp. 105-117.

Dand, I. (1988). Hydrodynamic Aspects of the Sinking of the Ferry Herald of Ferry Enterprise. Transactions of Royal Institute of Naval Architecture, Vol. 130, pp.145-167.

Domeh, V.D.K., Sobey, A.J. and Hudson, D.A. (2015). A preliminary experimental investigation into the influence of compartment permeability on damaged ship response in waves, Applied Ocean Research, Vol. 52, pp.27-36

Hearn, G. E. Lafforgue, D. Perdriset, E. and Saydan, D. (2008). The hydrodynamic and dynamic motion analysis of a damaged ship. Transactions of Royal Institute of Naval Architects, vol. 150, pp.75-99.

Li, Y. Sobey, A. J. and Tan, M. (2013). Investigation into the Effects of Petalling on Coefficient of Discharge during Compartment Flooding. Journal of Fluids and Structures, Vol. 45, pp.66-78.

Miller, D. (1978). Internal Flow Systems. BHRA Fluid Engineering.

Ruponen, P.(2006), Experimental Study on Progressive Flooding, Proceedings of Maritime Institute of Finland Seminar, Finland.

Ruponen, P. (2007). Progressive flooding of a damaged passenger ship. Helsinki Doctoral Thesis.

Ruponen, P. Kurvinen, P. Saisto, I. and Harras, J. (2010). Experimental and numerical study on progressive flooding in full-scale. Transactions of was found to give acceptable results; however, for longer wave periods this was found to over predict the flow rates and give larger free surface motion amplitudes due to ingress and egress.

\section{ACKNOWLEDGEMENTS}

The authors would like to thank the UK Ministry of Defence and Lloyd's Register EMEA for their support and without whom this research would not have been possible.

Royal Institution of Naval Architects, International Journal Maritime Engineering, A-197-A-207.

Smith, D. and Walker, W. J. (1923). Orifice flow. Proceedings of the Institute of Mechanical Engineers, vol. 104, pp.23-36.

Smith, T. W. P. (2009). Wave loading on damaged ships, University College London Doctoral Thesis.

Spouge, J. R. (1986). The Technical Investigation of the Sinking of the Ro-Ro Ferry European Gateway. Transactions of Royal Institute of Naval Architects, RINA, Vol. 128, pp. 49-72.

Suyuthi, A. Leira, B.J. and Riska, K. (2013). Fatigue damage of ship hulls due to local iceinduced stresses. Applied Ocean Research, vol. 42, pp.87-104.

Vassalos, D. and Turan, O. (1994). A Realistic Approach to Assessing the Damage Survivability of Passenger Ships. Transactions of Society of Naval Architects and Marine Engineer, SNAME, Vol. 102, pp. 367-394.

Wang, X. Li, A. Sobey, A.J. and Tan, M. (Under Review). Investigation into the effects of two immiscible fluids on coefficient of discharge during compartment flooding. Ocean Engineering.

Wood, C. D. (2013). Compartment Flooding and Motions of Damaged Ships. University of Southampton DoctoralThesis.

Wood, C. Hudson, D. \& Tan, M. (2010). CFD Simulation of Orifice Flow for the Flooding of Damaged Ships. Proceedings of the 12th Numerical Towing Tank Symposium. Duisberg, Germany. 


\begin{tabular}{|c|c|}
\hline Item & Description \\
\hline 1 & Top plate \\
\hline 2 & Bottom plate \\
\hline 3 & Tube \\
\hline 4 & Orifice plate \\
\hline 5 & Stand pipe \\
\hline 6 & Tie bar \\
\hline 7 & Cross beam \\
\hline 8 & Longitudinal beam \\
\hline 9 & Existing water tank \\
\hline 10 & Ball valve \\
\hline 11 & Schrader valve \\
\hline 12 & O ring \\
\hline 13 & O ring \\
\hline 14 & M5 $\times 10$ countersunk screw \\
\hline 15 & M8 x 25 screw \\
\hline 16 & M8 nut \\
\hline 17 & M10 nut \\
\hline 18 & M8 washer \\
\hline 19 & M10 washer \\
\hline 20 & M10 spring washer \\
\hline
\end{tabular}

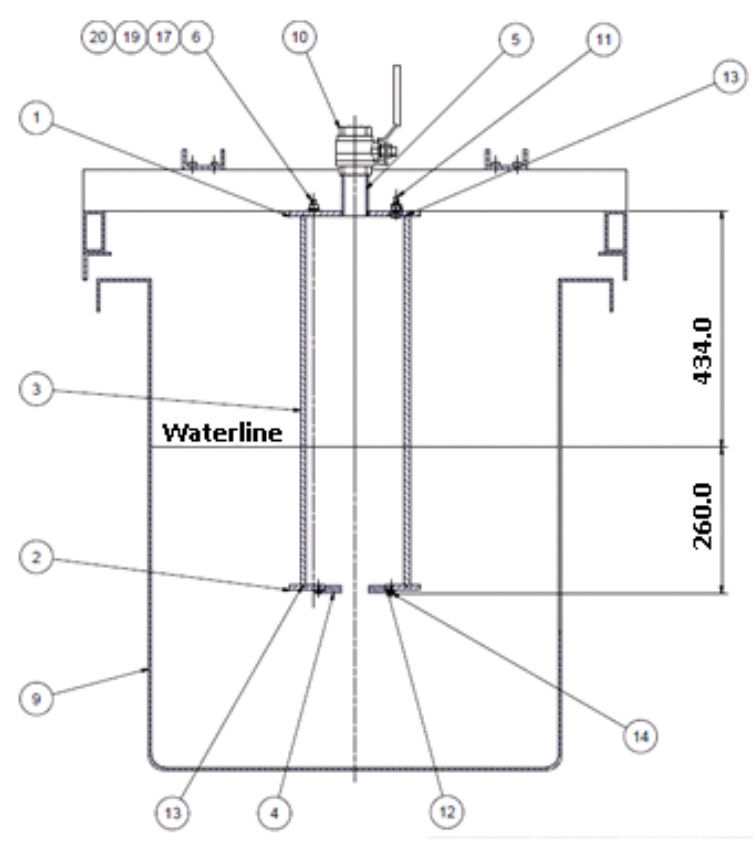

Figure 1: Transient flooding experimental equipment.

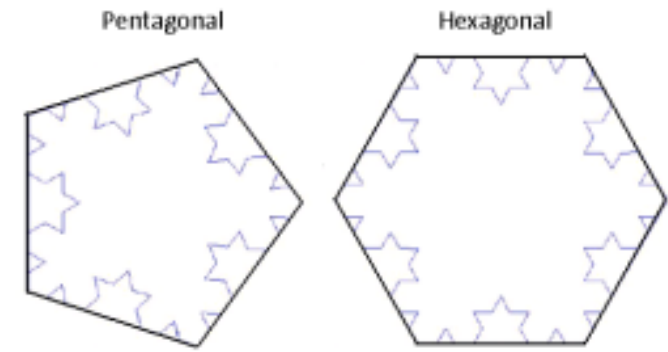

Figure 2: Examples of damage orifices given more realistic burring using fractals. 

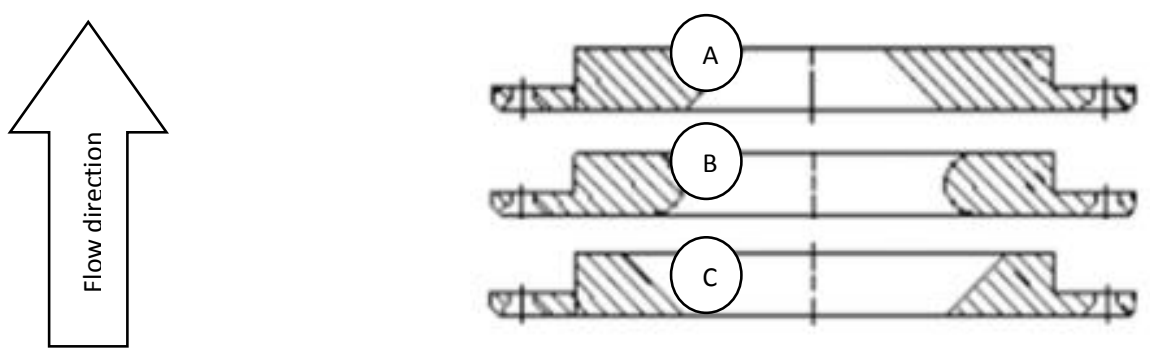

Figure 3: Side view of circular orifice edges, (A) convergent orifice section, (B) convergent-divergent orifice section and $(C)$ divergent nozzle section.

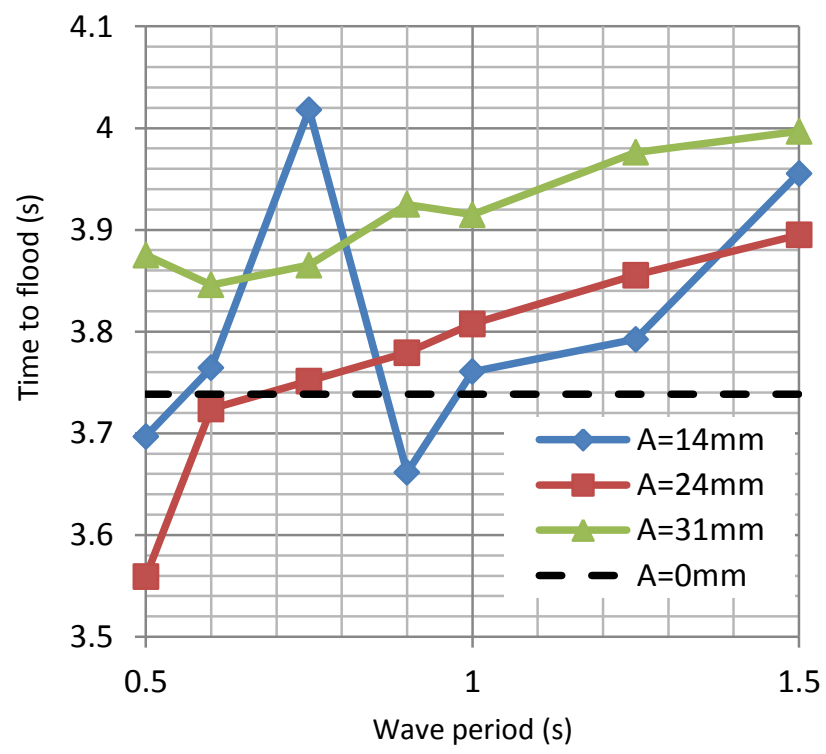

Figure 4: Averaged flooding time for varying wave amplitude and period for medium circle orifice. 


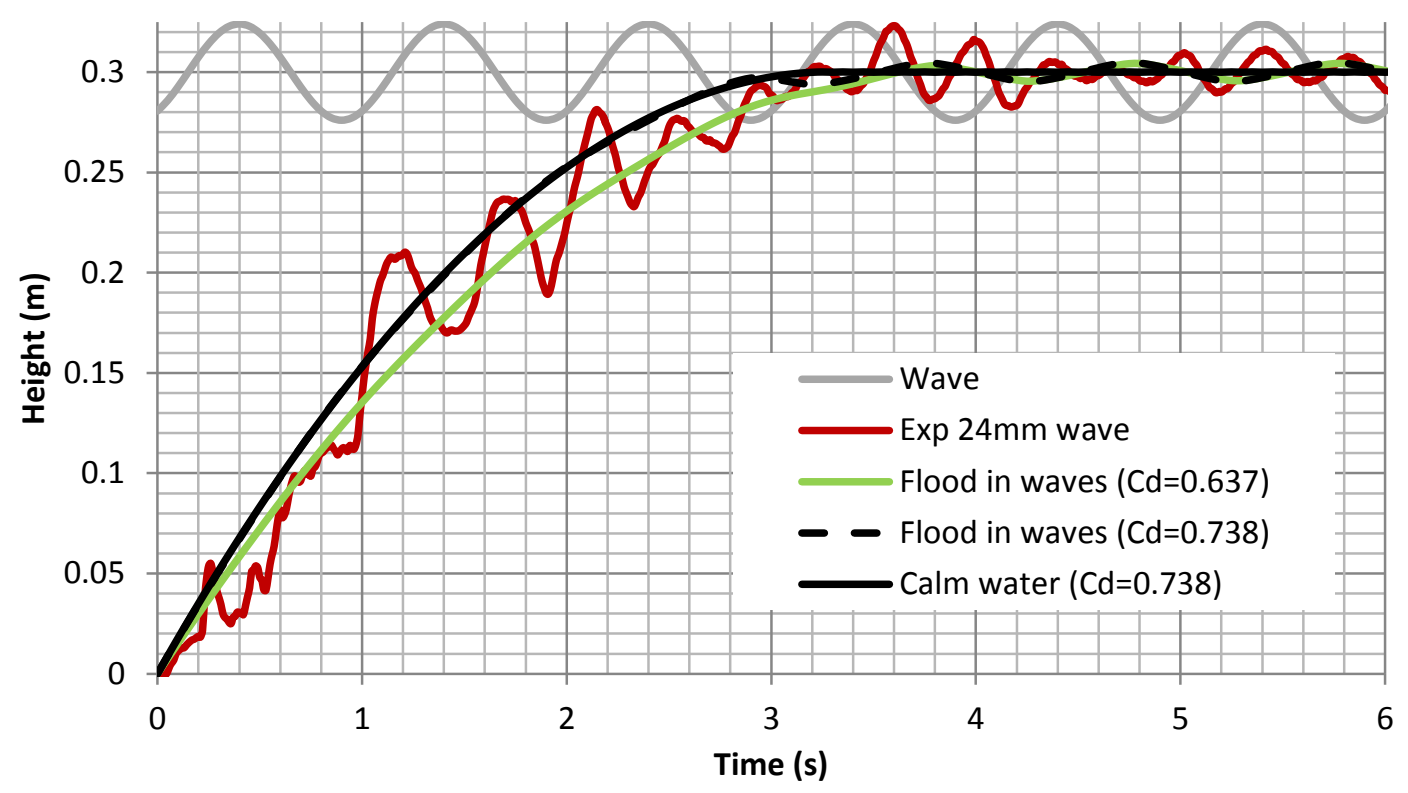

Figure 5: Comparison of calm and "in waves" models to experimental data

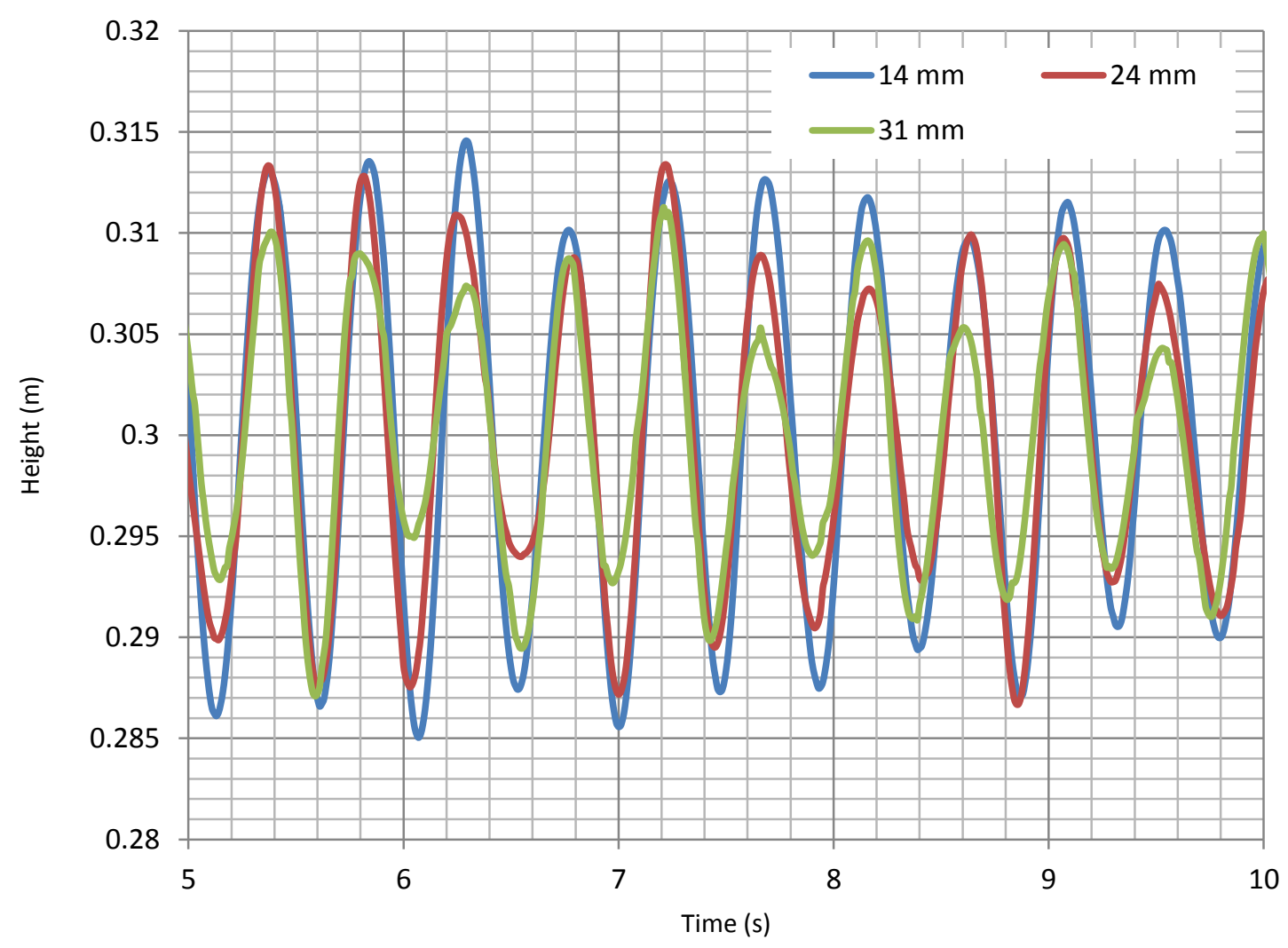

Figure 6: Experimental free surface height time histories in waves of varying amplitude 


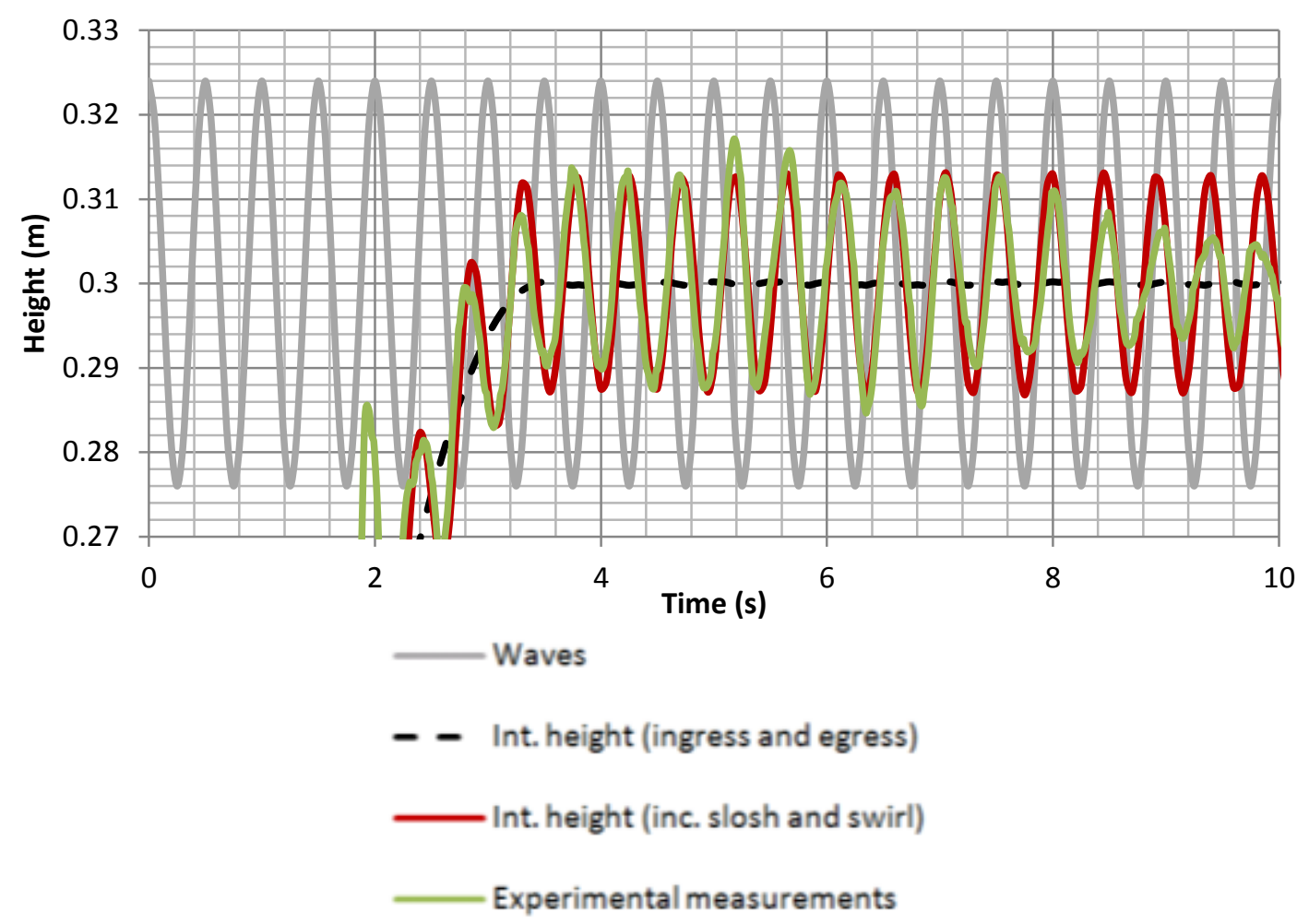

Figure 7: Free surface oscillations in waves of $T=0.5 \mathrm{~s}$; model compared to experiment.

Table 1: Results from irregular shape sensitivity.

\begin{tabular}{lccc}
\hline & Area $\left(\mathbf{m}^{\mathbf{2}}\right)$ & $\mathbf{T}_{\text {flood }}(\mathbf{s})$ & $\mathbf{C}_{\mathbf{d}}$ \\
\hline Small Circle & 0.001257 & 9.17 & 0.609 \\
Fractal S5 & 0.001257 & 11.59 & 0.481 \\
Fractal S6 & 0.001257 & 10.99 & 0.508 \\
Medium Circle & 0.002827 & 4.49 & 0.552 \\
Fractal M5 & 0.002827 & 4.61 & 0.538 \\
Fractal M6 & 0.002827 & 4.51 & 0.550 \\
Large Circle & 0.005027 & 2.80 & 0.498 \\
Fractal L5 & 0.005027 & 2.89 & 0.483 \\
Fractal L6 & 0.005027 & 2.83 & 0.494 \\
\hline
\end{tabular}


Table 2: Results table for the orifice edge sensitivity survey.

\begin{tabular}{cccccc}
\hline & Small Circle & Convergent & Medium Circle & Divergent & Rounded \\
\hline $\begin{array}{c}\text { Radius (m) } \\
\text { Minimum }\end{array}$ & 0.02 & 0.016 & 0.03 & 0.03 & 0.03 \\
$\begin{array}{c}\text { Area } \\
\left(\mathbf{x 1 0} \mathbf{~ m}^{-3}\right)\end{array}$ & 1.257 & 0.804 & 2.827 & 2.827 & 2.827 \\
$\mathbf{T}_{\text {flood }}(\mathbf{s})$ & 9.17 & 12.78 & 4.49 & 4.76 & 3.90 \\
$\mathbf{C}_{\mathbf{d}}$ & 0.609 & 0.682 & 0.552 & 0.521 & 0.636 \\
\hline
\end{tabular}

Table3: Results for orifice location sensitivity study (equivalent to small circle damage).

\begin{tabular}{cccc}
\hline $\begin{array}{c}\text { Displacement } \\
\text { from centre }\end{array}$ & Area $\left(\mathbf{m}^{2}\right)$ & $\mathbf{T}_{\text {flood }}(\mathbf{s})$ & $\mathbf{C}_{\mathbf{d}}$ \\
\hline $\mathbf{0 ~} \mathbf{~ m m}$ & 0.001257 & 9.17 & 0.609 \\
$\mathbf{1 3 . 7 5} \mathbf{~ m m}$ & 0.001257 & 9.06 & 0.616 \\
$\mathbf{2 7 . 5} \mathbf{~ m m}$ & 0.001257 & 9.47 & 0.589 \\
\hline
\end{tabular}

Table 4: Results for compartment flooding in waves.

\begin{tabular}{|c|c|c|c|c|c|c|c|c|c|}
\hline & \multicolumn{3}{|l|}{ Wave Amplitude $=\mathbf{1 4 m m}$} & \multicolumn{2}{|c|}{ Wave Amplitude = 24mm } & \multicolumn{2}{|c|}{ Wave Amplitude = 31mm } \\
\hline $\begin{array}{c}\text { Wave time } \\
\text { period (s) }\end{array}$ & $\begin{array}{c}\text { Average } \\
\text { flow rate } \\
\left(\mathbf{m}^{\mathbf{3}} \mathbf{s}^{-1}\right)\end{array}$ & $\begin{array}{c}\mathbf{T}_{\text {flood }} \\
(\mathbf{s})\end{array}$ & $\mathbf{C}_{\mathbf{d}}$ & $\begin{array}{c}\text { Average } \\
\text { flow rate } \\
\left(\mathbf{m}^{\mathbf{3}} \mathbf{s}^{-1}\right)\end{array}$ & $\begin{array}{c}\mathbf{T}_{\text {flood }} \\
(\mathbf{s})\end{array}$ & $\mathbf{C}_{\mathbf{d}}$ & $\begin{array}{c}\text { Average } \\
\text { flow rate } \\
\left(\mathbf{m}^{\mathbf{3}} \mathbf{s}^{-1}\right)\end{array}$ & $\begin{array}{c}\mathbf{T}_{\text {flood }} \\
(\mathbf{s})\end{array}$ & $\mathbf{C}_{\mathbf{d}}$ \\
\hline 0.5 & 0.002065 & 3.70 & 0.67 & 0.002145 & 3.56 & 0.70 & 0.00197 & 3.88 & 0.64 \\
\hline 0.6 & 0.002028 & 3.76 & 0.66 & 0.00205 & 3.72 & 0.67 & 0.001985 & 3.85 & 0.65 \\
\hline 0.75 & 0.0019 & 4.02 & 0.62 & 0.002035 & 3.75 & 0.66 & 0.001975 & 3.87 & 0.64 \\
\hline 0.9 & 0.002085 & 3.66 & 0.68 & 0.00202 & 3.78 & 0.66 & 0.001945 & 3.92 & 0.63 \\
\hline 1 & 0.00203 & 3.76 & 0.66 & 0.002005 & 3.81 & 0.65 & 0.00195 & 3.92 & 0.63 \\
\hline 1.25 & 0.002013 & 3.79 & 0.65 & 0.00198 & 3.86 & 0.64 & 0.00192 & 3.98 & 0.62 \\
\hline 1.5 & 0.00193 & 3.96 & 0.63 & 0.00196 & 3.90 & 0.64 & 0.00191 & 4.00 & 0.62 \\
\hline
\end{tabular}

